\title{
The Renin-Angiotensin Aldosterone System: Pathophysiological Role and Pharmacologic Inhibition
}

\author{
Steven A. Atlas, MD
}

\begin{abstract}
BACKGROUND: The renin-angiotensin aldosterone system (RAAS) is a hormonal cascade that functions in the homeostatic control of arterial pressure, tissue perfusion, and extracellular volume. Dysregulation of the RAAS plays an important role in the pathogenesis of cardiovascular and renal disorders.
\end{abstract}

OBJECTIVES: To review the role of the RAAS in the development of hypertensive cardiovascular disease and related conditions and provide an overview of the classes of pharmacologic agents that inhibit this system.

RESULTS: The RAAS is initiated by the regulated secretion of renin, the rate-limiting enzyme that catalyzes the hydrolysis of angiotensin (Ang) I from the $\mathrm{N}$-terminus of angiotensinogen. Ang I is in turn hydrolyzed by angiotensin-converting enzyme (ACE) to form Ang II, a potent vasoconstrictor and the primary active product of the RAAS. Recent evidence has suggested that other metabolites of Ang I and II may have biological activity, particularly in tissues. Development of agents that block the RAAS (e.g., beta blockers, ACE inhibitors [ACEls], and angiotensin receptor blockers [ARBs]) began as a therapeutic strategy to treat hypertension. Preclinical and clinical studies have indicated important additional cardiovascular and renal therapeutic benefits of ACEls and ARBs. However, blockade of the RAAS with these agents is incomplete.

CONCLUSION: Therapeutic approaches that target more complete inhibition of the RAAS may offer additional clinical benefits for patients with cardiovascular and renal disorders. These approaches may include dual blockade using ACEls and ARBs in combination, or new therapeutic modalities such as direct renin inhibition with aliskiren, recently approved for the treatment of hypertension.

KEYWORDS: Renin-angiotensin aldosterone system; Hypertension; Angiotensin II, renin; ACE inhibitors; Angiotensin receptor blockers

J Manag Care Pharm. 2007;13(8)(suppl S-b):S9-S20

Copyright@ 2007, Academy of Managed Care Pharmacy. All rights reserved.

\section{Author}

STEVEN A. ATLAS, MD, is an associate professor of medicine, Mount Sinai School of Medicine, New York, and chief, Cardiology/ Hypertension/Endocrinology Practice, and director, Hypertension Research Laboratory, James J. Peters VA Medical Center, Bronx, NY.

AUTHOR CORRESPONDENCE: Steven A. Atlas, MD,

130 W. Kingsbridge Rd., Bronx, NY 10468. Tel: (718) 584-9000;

Fax: (718) 741-4295; E-mail: steven.atlas@med.va.gov
7 he renin-angiotensin aldosterone system (RAAS) plays an integral role in the homeostatic control of arterial pressure, tissue perfusion, and extracellular volume. It functions as an unusual endocrine axis in which the active hormone, angiotensin (Ang) II, is formed in the extracellular space by sequential proteolytic cleavage of its precursors. This pathway is initiated by the regulated secretion of renin, the rate-limiting processing enzyme. Although renin was discovered more than a century ago, ${ }^{1}$ the significance of this system in the pathogenesis of cardiovascular and renal disorders has gained wide acceptance only during the past 3 decades, in large part because of the availability of specific pharmacologic agents that can block the system.

In this article, I will review the evidence concerning the role of the RAAS in the development of hypertensive cardiovascular disease and related conditions and provide an overview of the agents that inhibit this system.

\section{Historical Perspective}

In 1898, Tigerstedt and Bergmann published an account of their research demonstrating the existence of a heat-labile substance in crude extracts of rabbit renal cortex that caused a sustained increase in arterial pressure. ${ }^{1}$ They proposed the term "renin" for a presumed humoral pressor agent secreted by the kidney, a concept that was widely disputed or ignored until the classical studies of Goldblatt and colleagues, published in 1934, that showed that renal ischemia induced by clamping of the renal artery could induce hypertension." Shortly thereafter it was shown that the ischemic kidney also released a heat-stable, short-lived pressor substance, in addition to renin. This finding eventually led to the recognition that renin's pressor activity was indirect and resulted from its proteolytic action on a plasma substrate (eventually termed "angiotensinogen") to liberate a direct-acting pressor peptide. This peptide was initially termed "angiotonin" or "hypertensin" by competing investigators in the United States (Page and colleagues) and in Argentina (Braun-Menendez and colleagues), who ultimately compromised on the term "angiotensin." In the early 1950s, during attempts at purification, Skeggs and colleagues discovered that this peptide existed in 2 forms, eventually termed Ang I and II. ${ }^{2}$ In later work, they demonstrated that Ang I was cleaved by a contaminating plasma enzyme, termed "angiotensin-converting enzyme," to generate the active pressor peptide Ang II. ${ }^{3}$ Soon after, the work of several investigators, including Laragh, Genest, Davis, Ganong, and their colleagues, culminated in the discovery that Ang II also stimulated the release of the adrenal cortical hormone aldosterone, a major 
regulator of sodium and potassium balance. ${ }^{4}$ These landmark discoveries established the concept that a single system, the RAAS, was involved in the regulation of both blood pressure and fluid and electrolyte balance.

\section{Components of the RAAS}

The renin-angiotensin aldosterone hormonal cascade begins with the biosynthesis of renin by the juxtaglomerular cells (JG) that line the afferent (and occasionally efferent) arteriole of the renal glomerulus. Renin is synthesized as a preprohormone, and mature (active) renin is formed by proteolytic removal of a 43-amino-acid prosegment peptide from the N-terminus of prorenin, the proenzyme or renin precursor. Mature renin is stored in granules of the JG cells and is released by an exocytic process involving stimulus-secretion coupling into the renal and then the systemic circulation. In addition to this regulated pathway, it appears that the kidney also releases unprocessed prorenin via a constitutive pathway. In fact, prorenin accounts for about 70\% to $90 \%$ of the immunoreactive renin in the human circulation. The potential biological significance of this finding is poorly understood at present.

Active renin secretion is regulated principally by 4 interdependent factors: (1) a renal baroreceptor mechanism in the afferent arteriole that senses changes in renal perfusion pressure, (2) changes in delivery of $\mathrm{NaCl}$ (sensed as changes in $\mathrm{Cl}^{-}$concentration) to the macula densa cells of the distal tubule (which lie close to the JG cells and, together, form the "JG apparatus"), (3) sympathetic nerve stimulation via beta-1 adrenergic receptors, and (4) negative feedback by a direct action of Ang II on the JG cells. ${ }^{5}$ Renin secretion is stimulated by a fall in perfusion pressure or in $\mathrm{NaCl}$ delivery and by an increase in sympathetic activity. Renin is also synthesized in other tissues, including brain, adrenal gland, ovary, and visceral adipose tissue, and perhaps heart and vascular tissue. The factors regulating synthesis and possible actions of renin in these other tissues are poorly understood.

Control of renin secretion is a key determinant of the activity of the RAAS. Renin regulates the initial, rate-limiting step of the RAAS by cleaving the N-terminal portion of a large molecular weight globulin, angiotensinogen, to form the biologically inert decapeptide Ang I or Ang-(1-10), as shown in the Figure. The primary source of systemic circulating angiotensinogen is the liver, but angiotensinogen mRNA expression has also been detected in many other tissues, including kidney, brain, heart, vascular, adrenal gland, ovary, placenta, and adipose tissue. ${ }^{6}$ Angiotensinogen is secreted constitutively by the liver, so plasma levels are generally stable and do not change acutely; however, both hepatic and extrahepatic synthesis have been shown to rise in response to glucocorticoids, estrogens and other sex steroids, thyroid hormone, inflammatory cytokines (e.g., interleukin-1 and tumor necrosis factor), and Ang II. ${ }^{6}$ Adrenal insufficiency, orchiectomy, hypothyroidism, and insulin deficiency have been associated with a decline in plasma concentration and/or tissue mRNA expression of angiotensinogen. ${ }^{6}$ Long-term elevations in angiotensinogen concentration, which occur with pregnancy, Cushing's syndrome, or glucocorticoid treatment, may be a risk factor for hypertension, although there is evidence that chronic stimulation of angiotensinogen may be partly compensated for by a reduction in renin secretion.

The inactive decapeptide Ang I is hydrolyzed by angiotensin-converting enzyme (ACE), which removes the C-terminal dipeptide to form the octapeptide Ang II [Ang-(1-8)], a biologically active, potent vasoconstrictor. ACE is a membrane-bound exopeptidase and is localized on the plasma membranes of various cell types, including vascular endothelial cells, microvillar brush border epithelial cells (e.g., renal proximal tubule cells), and neuroepithelial cells. It is this membrane-bound ACE that is thought to be physiologically important. ACE also exists in a soluble form in plasma, but this form may simply reflect turnover and clearance of membrane-bound ACE. ACE (also known as kininase II) metabolizes a number of other peptides, including the vasodilator peptides bradykinin and kallidin, to inactive metabolites. ${ }^{7}$ Thus, functionally, the enzymatic actions of ACE potentially result in increased vasoconstriction and decreased vasodilation.

Although Ang II is the primary active product of the RAAS, there is evidence that other metabolites of Ang I and II may have significant biological activity, particularly in tissues. Ang III and IV are formed by the sequential removal of amino acids from the N-terminus of Ang II by the action of aminopeptidases (Figure). They are most likely produced in tissue with high levels of aminopeptidases A and N, such as brain and kidney tissue. ${ }^{8}$ Ang III [Ang-(2-8)], a heptapeptide formed by removal of the first N-terminal amino acid, is present in the central nervous system (CNS), where it is thought to play an important role in tonic blood pressure maintenance and in hypertension. ${ }^{8}$ Ang IV [Ang-(3-8)] is a hexapeptide formed by further enzymatic degradation of Ang III. ${ }^{8}$ Preclinical studies have suggested a cooperative effect of Ang IV in Ang II signaling. For instance, it appears that in the brain, Ang IV increases blood pressure by cooperating with Ang II on angiotensin II type $1\left(\mathrm{AT}_{1}\right)$-receptor signaling (see below), because its hemodynamic effects require the presence of both Ang II and functional $\mathrm{AT}_{1}$ receptors. ${ }^{8}$

Peptides truncated at the C-terminus of Ang II may also have biological activity. For example, Ang-(1-7), a heptapeptide fragment of Ang II, can be formed from Ang I or Ang II by the actions of several endopeptidases or from Ang II by the action of carboxypeptidases, including one with significant structural homology to ACE (which has been termed "ACE 2"). Unlike ACE, this enzyme does not convert Ang I to Ang II and its activity is not affected by ACE inhibitors (ACEIs). ${ }^{7}$ Ang-(1-7), which appears to act via a unique receptor (see below), was first described to have vasodilatory effects and act as a natural ACEI. Cardioprotective effects have also been proposed to result from a direct effect of Ang-(1-7) 


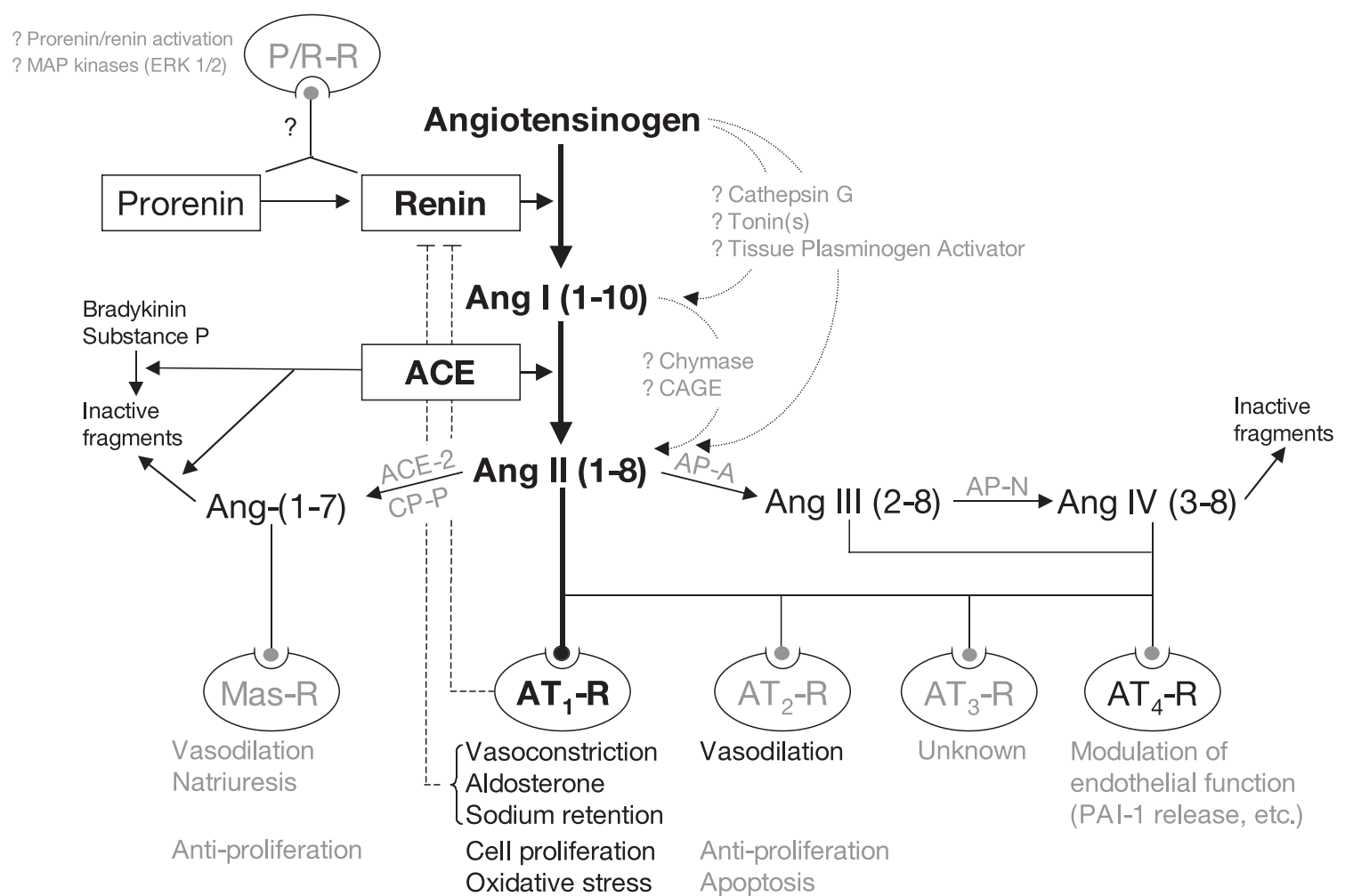

The classical RAAS pathway is highlighted in boldface type. Renin, normally secreted in response to underperfusion of the kidneys (not shown), cleaves the decapeptide Ang I from angiotensinogen, and Ang I is converted to Ang II by ACE. The dashed lines indicate feedback inhibition of renin secretion, which occurs both via a direct AT 1 receptor mediated action of Ang II ("short loop") and via AT 1 -mediated restoration of blood pressure and volume ("long loop"). Other pathways that are speculative or of unproven physiological significance in vivo are depicted in light text. Ang II can be converted to Ang-(1-7) by ACE 2 or other carboxypeptidases. Ang II can also be cleaved by aminopeptidases to form Ang III and Ang IV. These peptides exert their biological effects by binding to various subtypes of angiotensin receptors. In addition, Ang-(1-7) can be formed directly from Ang I by the action of endopeptidases (not shown), and further metabolism of peptides to inactive fragments involves several amino-, carboxy-, and endopeptidases. A number of other proteolytic enzymes are shown that potentially can contribute to Ang I or Ang II synthesis. Lastly, both renin and prorenin may exert direct cellular actions by binding to a specific prorenin/renin receptor. ACE=angiotensin-converting enzyme; Ang=angiotensin; AP-A/AP-N=aminopeptidase A/aminopeptidase N; $A T-R=$ angiotensin receptor subtype; $C A G E=$ chymostatin-sensitive angiotensin II-generating enzyme; $C P-P=$ carboxypeptidase $P ; M A P=$ mitogen-activated protein; Mas- $R=$ Mas receptor; $P / R-R=$ prorenin/renin receptor; $P A I-1=$ plasminogen activator inhibitor-1.

on heart cells or a generalized systemic effect, ${ }^{8}$ but evidence for such actions in human studies is lacking. ACE 2 can also cleave a single amino acid from the C-terminus of Ang I to form Ang-(1-9), a peptide with no known function at this time.

As already noted, Ang II is the primary effector of a variety of RAAS-induced physiological and pathophysiological actions. At least 4 angiotensin receptor subtypes have been described. ${ }^{9}$ The type $1\left(\mathrm{AT}_{1}\right)$ receptor mediates most of the established physiological and pathophysiological effects of Ang II (Figure). These include actions on the cardiovascular system (vasoconstriction, increased blood pressure, increased cardiac contractility, vascular and cardiac hypertrophy), kidney (renal tubular sodium reabsorption, inhibition of renin release), sympathetic nervous system, and adrenal cortex (stimulation of aldosterone synthesis). ${ }^{7}$

The $\mathrm{AT}_{1}$ receptor also mediates effects of Ang II on cell growth and proliferation, inflammatory responses, and oxidative stress. ${ }^{2}$ This receptor, which is typical of the G protein-coupled receptor superfamily containing 7 membrane-spanning sequences, is widely distributed on many cell types in Ang II target organs.

The type $2\left(\mathrm{AT}_{2}\right)$ receptor is abundant during fetal life in the brain, kidney, and other sites, and its levels decrease markedly in the postnatal period. There is some evidence that, despite low levels of expression in the adult, the $\mathrm{AT}_{2}$ receptor might mediate vasodilation and antiproliferative and apoptotic effects 
in vascular smooth muscle and inhibit growth and remodeling in the heart. ${ }^{79}$ In the kidney, it has been proposed that activation of $\mathrm{AT}_{2}$ receptors may influence proximal tubule sodium reabsorption and stimulate the conversion of renal prostaglandin $\mathrm{E}_{2}$ to prostaglandin $\mathrm{F}_{2 \alpha} \cdot{ }^{2,7}$ However, the importance of any of these $\mathrm{AT}_{2}$-mediated actions remains uncertain.

The type $4\left(\mathrm{AT}_{4}\right)$ receptors are thought to mediate the release of plasminogen activator inhibitor 1 by Ang II and by the N-terminal truncated peptides (Ang III and Ang IV), but the function of the type $3\left(\mathrm{AT}_{3}\right)$ receptors is unknown. ${ }^{9}$ Lastly, the putative effects attributed to the C-terminal truncated peptide Ang 1-7, including vasodilatation, natriuresis, antiproliferation, and cardiac protection, are presumed to be mediated by a unique receptor that does not bind Ang II, most likely a product of the Mas proto-oncogene known as the Mas receptor. ${ }^{9}$

In addition to receptors for the angiotensin peptides, very recent evidence suggests the existence of high-affinity cell surface receptors that bind both renin and prorenin in several tissues, including heart, brain, placenta, and kidney, with localization to glomerular mesangium and subendothelial vascular smooth muscle. ${ }^{10}$ One receptor that has been carefully characterized has been reported to cause reversible activation of bound prorenin and to enhance the catalytic activity of bound renin, thus serving as a template for local Ang I generation. The receptor also has been reported to initiate intracellular signaling, independent of Ang peptide synthesis, leading to activation of mitogen-activated protein kinases ERK1 and ERK2 (Figure). ${ }^{10}$

These findings raise the possibility of Ang II-independent effects on cellular growth responses by renin or prorenin. An intriguing series of studies raises the possibility that such putative receptor-mediated mechanisms may contribute to the development of experimental diabetic nephropathy. ${ }^{11}$ Research into this new aspect of RAAS biology is progressing at a rapid pace, although there is as yet no evidence for human counterparts to these novel mechanisms.

As already noted, Ang II, via the $\mathrm{AT}_{1}$ receptor, also stimulates the production of aldosterone by the zona glomerulosa, the outermost zone of the adrenal cortex. Aldosterone is a major regulator of sodium and potassium balance and thus plays a major role in regulating extracellular volume. It enhances the reabsorption of sodium and water in the distal tubules and collecting ducts (as well as in the colon and salivary and sweat glands) and thereby promotes potassium (and hydrogen ion) excretion. ${ }^{12}$ Ang II, together with extracellular potassium levels, are the major regulators of aldosterone, but Ang II synthesis may also be stimulated by adrenocorticotrophic hormone (ACTH; corticotropin), norepinephrine, endothelin, and serotonin and inhibited by atrial natriuretic peptide and nitric oxide (NO). It is also important to note that Ang II is a major trophic factor for the zona glomerulosa, which can atrophy (reversibly) in its absence.

\section{Classical Endocrine Pathway of Angiotensin Biosynthesis}

The classical enzymatic cascade of the RAAS is depicted in the Figure. Reduction in renal perfusion pressure and afferent arteriolar pressure induced, for instance, by a reduction in circulating blood volume triggers the release of renin from the stretchsensitive JG cells lining mainly the afferent renal arterioles. This mechanism is reinforced by a reduction in the tubular fluid sodium chloride concentration as sensed by the macula densa cells of the distal tubules and at times by an increase in sympathetic discharge to the kidney. In the extracellular space, renin cleaves the N-terminal 10 amino acids of circulating angiotensinogen to form the biologically inert decapeptide Ang I, which in turn is converted to the active octapeptide Ang II by removal of the C-terminal dipeptide by ACE. As mentioned earlier, this last step in Ang II biosynthesis is mediated mainly by membranebound ACE, which is nearly ubiquitous on vascular endothelium. The concept of a circulating endocrine cascade has been frequently misinterpreted to imply that Ang II is a circulating "hormone," but this is in fact unlikely. Instead, both Ang I and Ang II, which have very short half-lives, are probably synthesized very close to their site of action, with renin serving as the circulating hormonal signal that initiates the pathway at local sites.

The diverse actions of Ang II mediated by the $\mathrm{AT}_{1}$ receptor play a key role in restoring or maintaining circulatory homeostasis. In addition to stimulating the production (and release) of aldosterone from the adrenal cortex, Ang II promotes the constriction of renal and systemic arterioles and the reabsorption of sodium in proximal segments of the nephron. The increase in blood pressure and volume, resulting from the effects of Ang II and aldosterone on their target organs, serves to restore renal perfusion and thereby inhibits further release of renin. Although the RAAS thus plays an important role in normal circulatory homeostasis, continued or inappropriate activation of the system is thought to contribute to the pathophysiology of diseases such as hypertension and heart failure.

\section{Tissue RAAS and Alternative Pathways of Angiotensin Biosynthesis}

The evidence that angiotensin synthesis can occur in several tissues as well as the circulation, together with the characterization of several subtypes of angiotensin receptors and signal transduction pathways, the identification of truncated angiotensin peptides with possible unique actions, and most recently, the identification of putative cell surface receptors for renin and prorenin, has resulted in expansion of the traditional circulating RAAS paradigm to include the so-called "tissue RAAS." The prevailing concept is that the RAAS functions both as a circulating system and as a tissue paracrine/autocrine system., ${ }^{7,13}$

There is evidence that local or "tissue" Ang II biosynthesis may be initiated by renin and/or angiotensinogen taken up from the circulation. In addition, independent Ang II 
generating systems have been postulated to exist in the heart, peripheral blood vessels, kidney, brain, adrenal glands, pituitary, adipose tissue, testes, ovaries, and skin. ${ }^{7,13}$ Serine proteases, including several kallikrein-like enzymes (tonins), cathepsin $G$, and chymase are thought to contribute to Ang II formation in the tissue RAAS. ${ }^{14}$ Studies have suggested that non-ACE pathways are, by inference, responsible for about $40 \%$ of Ang II generation in the intact human kidney ${ }^{15}$ and that chymase is the dominant Ang II-generating pathway in the human heart, coronary arteries, and atherosclerotic aorta in vitro. ${ }^{14,16,17}$ It has thus been proposed that abnormal activation of the tissue RAAS may contribute to the pathogenesis of cardiovascular disease even in the absence of derangements in the circulating system. ${ }^{18}$ It must be considered, however, that the bulk of the evidence favoring alternate enzymatic pathways in the synthesis of angiotensin peptides comes from in vitro or indirect observations, so that such concepts remain speculative at present.

Under physiological conditions, the apparent function of the cardiac RAAS is to maintain cellular balance of inhibiting and inducing cell growth, and proliferation and mediation of adaptive responses to myocardial stretch. ${ }^{19}$ The majority of Ang II in cardiac tissue appears to be produced by local synthesis of Ang I and subsequent local conversion to Ang II, rather than from uptake of peptides from the systemic circulation. ${ }^{7}$ Although it has been suggested that locally synthesized renin and/or additional proteolytic enzymes may be involved in this synthetic process, current evidence favors the concept that circulating renin and angiotensinogen, which are able to pass through the endothelial barrier, are taken up by cardiac tissue where they act locally. ${ }^{20}$ Ang II exerts an inotropic effect (at least in atrial preparations), mediates myocyte hypertrophy via the $\mathrm{AT}_{1}$ receptor, and is involved in cardiac remodeling. ${ }^{19}$ Pathologic activation of cardiac RAAS, perhaps through local upregulation of ACE levels, has been proposed to contribute to the development and maintenance of left ventricular hypertrophy. ${ }^{18}$

Vascular smooth muscle, endothelial, and endocardial cells generate Ang I and Ang II, again apparently via the uptake of circulating renin. ${ }^{7}$ It has been suggested that the vascular RAAS contributes to the maintenance of cardiovascular homeostasis through its effects on both $\mathrm{AT}_{1}$ and $\mathrm{AT}_{2}$ receptors and mediates long-term effects on vascular remodeling by stimulating proliferation of vascular smooth muscle cells and fibroblasts. ${ }^{19}$ Endothelial dysfunction is associated with upregulation of local tissue ACE, which might contribute to disrupting the balance of vasodilation and vasoconstriction. Activation of vascular ACE may also alter other functions, including vascular smooth muscle cell growth and the inflammatory and oxidative state of the vessel wall. ${ }^{18}$ In addition, the production of reactive oxidative species (superoxide and hydrogen peroxide), which is enhanced by Ang II, has been associated with inflammation, atherosclerosis, hypertrophy, remodeling, and angiogenesis. ${ }^{19}$
The intrarenal RAAS may explain the primary role of Ang II as a paracrine substance in the control of renal function. The direct intrarenal actions of Ang II include renal vasoconstriction, tubular sodium reabsorption, sensitivity of tubuloglomerular feedback, modulation of pressure-natriuresis, and promotion of renal tissue growth. ${ }^{7,13}$ Under normal conditions, Ang II constricts both the afferent and efferent arterioles and stimulates mesangial cell contraction, which results in reduced renal blood flow, glomerular filtration rate (GFR), and filtered sodium load. ${ }^{7}$ On the one hand, overactivation of the intrarenal RAAS may thus contribute to the pathophysiology of sodium-retaining states, such as hypertension and congestive heart failure (CHF). ${ }^{7}$ On the other hand, in conditions characterized by severe impairment of renal perfusion, such as renal artery stenosis, the afferent circulation, which is dilated as a result of autoregulation, is relatively refractory to the constrictive actions of Ang II, and the predominant constriction of efferent arterioles by Ang II plays a major role in maintaining glomerular perfusion pressure and, thus, GFR.

Although systemic Ang II may affect CNS function at selected sites, the brain is largely isolated from the circulating RAAS by the blood-brain barrier. Therefore, local Ang II synthesis by a brain RAAS has been proposed to play a role in central blood pressure regulation. ${ }^{19}$ Increases in brain renin activity, renin and angiotensinogen mRNA, and detectable numbers of $\mathrm{AT}_{1}$ - and $\mathrm{AT}_{2}$-receptor subtypes have been reported in hypertensive rats. ${ }^{19}$ Selective inhibition of brain $\mathrm{AT}_{1^{-}}$and $\mathrm{AT}_{2}$-receptors has been shown to lower blood pressure in hypertensive rats. ${ }^{13}$ Furthermore, direct administration of Ang II into the brain has been shown to increase blood pressure $e^{13,19}$ as a result of the combined effects of vasopressin release, sympathetic nervous system activation, and inhibition of baroreflexes. ${ }^{13}$ Studies in transgenic rats with permanent inhibition of brain angiotensinogen synthesis have demonstrated significantly lower systolic blood pressure compared with controls. ${ }^{19}$

All components of the RAAS are present in adrenal cortex and comprise the adrenal RAAS. Renin and angiotensinogen mRNA have been identified in the adrenal gland, and Ang II formation has been demonstrated in zona glomerulosa cells. ${ }^{7}$ Most (90\%) adrenal renin activity has been localized to the zona glomerulosa, ${ }^{7}$ and more than $90 \%$ of adrenal Ang II originates at local tissue sites. ${ }^{21}$ In transgenic animal models it has been shown that sodium restriction can increase adrenal renin and aldosterone independently of plasma or kidney renin concentrations. Additionally, bilateral nephrectomy, which decreases cardiac and vascular renin, does not decrease adrenal renin in experimental animals.,21 These findings support the concept of kidney-independent renin (and thus, Ang II) production in the adrenal glands. It is not known if the adrenal RAAS functions as a paracrine or autocrine system or if it has a pathophysiologic role, and the relative importance of systemic versus locally synthesized Ang II in the control of adrenal function is uncertain. 
Generally speaking, it is thought that the physiologic role of tissue RAAS is complementary to the classical circulating RAAS and serves as a mechanism for longer-term maintenance of balance or homeostasis at the tissue level between opposing effects mediated by the system (e.g., growth promotion and inhibition in the heart and vasculature) ${ }^{19}$ Pathophysiologic processes might hypothetically occur when components of the RAAS are overexpressed or inhibited, thus disturbing the intricate balance of this regulatory system.

\section{Dysregulation of the RAAS in Cardiovascular Disorders}

Dysregulation of the RAAS is involved in the pathogenesis of several hypertensive disorders (Table). ${ }^{22-24}$ It should be noted that RAAS dysregulation in clinical hypertensive disorders has been conceptualized at the level of the classical circulating RAAS, and the potential contributions of tissue RAAS dysregulation remain poorly defined.

In addition to RAAS involvement in secondary forms of hypertension, there is evidence that perturbations of the RAAS are involved in essential hypertension as well as in the responses of cardiovascular and renal tissue to hypertensive and nonhypertensive injury. It is established that plasma renin levels vary widely in patients with "essential" hypertension (Table). ${ }^{22}$ Approximately 15\% of patients with essential hypertension have mild to moderate increases in plasma renin activity (PRA), with several postulated mechanisms, including increased sympathetic activity and mild volume depletion. Such high-renin essential hypertension is particularly prevalent among younger males. The majority (50\% to 60\%) of essential hypertensive patients have PRA within the "normal" range, although it has been argued that a normal renin level in the face of hypertension (which ought to suppress renin secretion) may be inappropriate.

Therapeutic responses to RAAS blocking agents indicate that maintenance of normal renin levels may indeed contribute to blood pressure elevation, suggesting that renin-dependent mechanisms may be involved in more than $70 \%$ of patients with essential hypertension. On the other hand, about 25\% to 30\% have evidence of low or suppressed renin levels, a finding that may be an expected response or that may, in some cases, reflect, by analogy to primary aldosteronism, sodium or volume excess (so-called "volume-dependent" hypertension). Low-renin hypertension is more common among older people with hypertension, women, African Americans, and patients with type 2 diabetes, as well as among patients with chronic renal parenchymal disease.

Although such patients often have lesser blood pressurelowering benefit from RAAS blocking agents, there is evidence that the circulating levels of PRA might not necessarily reflect tissue activities of the system. This is particularly evident with regard to the kidney, with several lines of evidence pointing to substantial involvement of intrarenal Ang II in progression of renal damage (and substantial benefit of RAAS blockade), despite low circulating levels of renin and Ang II.
The RAAS also plays a pivotal role in several nonhypertensive conditions, and in particular in CHF and the other edematous disorders (cirrhosis with ascites and the nephrotic syndrome). In these conditions, all characterized by underperfusion of the kidneys due to reduced "effective arterial volume," secondary hypersecretion of renin leads to secondary aldosteronism, which makes an important contribution to progressive edema. In addition, with regard to heart failure, the contribution of Ang II to increased peripheral vascular resistance (cardiac afterload) also plays a major role in progressive ventricular dysfunction.

Beyond progression of renal disease, there is additional clear evidence (again from responses to RAAS blockade) of involvement of Ang II in development of both vascular and cardiac hypertrophy and remodeling, as well as on mechanisms that contribute to vascular damage and atherosclerosis, effects that appear to have major impact on morbidity and mortality. Unlike the case for secondary hypertensive syndromes where the nature of RAAS dysregulation is well defined (Table), the reasons for these pathologic effects of Ang II are uncertain since they often occur in the absence of any perturbation of the circulating RAAS. It has been inferred that there may be dysregulation of some component(s), such as of ACE levels, the balance of AT-receptor subtypes, or even local synthesis of renin or angiotensinogen, to account for such phenomena, but clearcut evidence of such derangements are mostly lacking. It also remains possible that the RAAS plays a "passive" role in such events-that is, that tissue injury can be accelerated even in the presence of "normal" Ang II levels.

\section{RAAS Inhibition}

\section{Early Preclinical Findings}

Because renin is the initial and rate-limiting step in the RAAS cascade, it has long been considered the logical therapeutic target for blocking the system. Preclinical studies with antirenin antibodies and then with early synthetic renin inhibitors established the potential utility of RAAS inhibition. In these studies, renin inhibition induced decreases in plasma renin levels (generally measured in these early studies as plasma renin activity or PRA), Ang I, Ang II, and aldosterone, along with decreases in blood pressure. ${ }^{25}$ These studies also provided evidence that blood pressure-lowering activity was due to inhibition of PRA. ${ }^{25}$ However, pharmacologic activity of the early renin inhibitors could only be achieved with intravenous infusion, and the development of an orally active direct renin inhibitor was fraught with numerous difficulties arising from issues of potency, low bioavailability, duration of action, and costs of synthesis. As a result, further development of these agents was halted in the mid-1990s. Concurrently, other strategies for inhibiting the RAAS progressed to clinical use. 


\section{TABLE Hypertensive Disorders Involving Dysregulation of the Renin-Angiotensin Aldosterone System ${ }^{22-24}$}

\begin{tabular}{|c|c|c|}
\hline Hypertensive Disorders & RAAS Dysregulation & Clinical Manifestations \\
\hline Renin-secreting neoplasms & $\begin{array}{l}\text { Primary hypersecretion of renin by renal } \\
\text { hemangiopericytomas ("JG cell tumors"), } \\
\text { some Wilms' tumors and renal and } \\
\text { extrarenal carcinomas (ovary, pancreas, lung) } \\
\text { Secondary aldosteronism }\end{array}$ & $\begin{array}{l}\text { Severe renin-dependent hypertension (accelerating hypertension } \\
\text { common) } \\
\text { Hypokalemia; sodium retention limited by pressure natriuresis }\end{array}$ \\
\hline $\begin{array}{l}\text { Renovascular hypertension } \\
\text { Atheromatous (main) } \\
\text { Fibromuscular (main or branch) } \\
\text { Renal emboli/segmental infarcts } \\
\text { Renal artery aneurysms } \\
\text { Renal artery dissection/injury } \\
\text { Subcapsular hematoma }\end{array}$ & $\begin{array}{l}\text { Hypersecretion of renin due to segmental, } \\
\text { unilateral, or bilateral renal ischemia }\end{array}$ & $\begin{array}{l}\text { Hypertension (usually renin-dependent, accelerating hypertension } \\
\text { common); progressive renal dysfunction possible } \\
\text { Sodium retention (depending on extent of renal compromise); } \\
\text { hypokalemia may be masked by decreased distal delivery of sodium }\end{array}$ \\
\hline $\begin{array}{l}\text { Malignant hypertension and other } \\
\text { renal small vessel disease (e.g., } \\
\text { polyarteritis, scleroderma, } \\
\text { hemolytic uremic syndrome, lupus) }\end{array}$ & $\begin{array}{l}\text { Hypersecretion of renin due to generalized } \\
\text { renal ischemia } \\
\text { Secondary aldosteronism }\end{array}$ & $\begin{array}{l}\text { Severe renin-dependent hypertension } \\
\text { Renal dysfunction } \\
\text { Microangiopathic hemolytic anemia } \\
\text { Hypertensive encephalopathy } \\
\text { Sodium retention and hypokalemia variable (as above) }\end{array}$ \\
\hline $\begin{array}{l}\text { Pheochromocytoma and other } \\
\text { catecholamine-secreting tumors }\end{array}$ & $\begin{array}{l}\text { Hypersecretion of renin due to } \\
\text { catecholamine excess } \\
\text { Renal ischemia due to extrinsic renal } \\
\text { compression and/or neurofibromatosis } \\
\text { involving the renal artery (occasionally) } \\
\text { Secondary aldosteronism }\end{array}$ & $\begin{array}{l}\text { Sustained or paroxysmal hypertension (accelerating hypertension } \\
\text { common) } \\
\text { Other typical manifestations of catecholamine excess } \\
\text { Hypokalemia; sodium retention often limited by pressure } \\
\text { natriuresis }\end{array}$ \\
\hline Essential hypertension & $\begin{array}{l}\text { HR ( 15\%)_PRA mildly elevated, } \\
\text { cause uncertain (sympathetic drive?, } \\
\text { hypovolemia?) } \\
\text { NR ( } 60 \%) \text { _PRA within "normal" range, } \\
\text { but may be inappropriate in setting of } \\
\text { hypertension } \\
\text { LR ( 25\%)_PRA low due to several possible } \\
\text { mechanisms (sodium/volume excess?, } \\
\text { nephrosclerosis?, appropriate response?) }\end{array}$ & $\begin{array}{l}\text { Hypertension usually responds well to RAAS blockade } \\
\text { Increased susceptibility to heart attack or stroke? } \\
\text { Hypertension often responds to RAAS blockade } \\
\text { Hypertension responds best to diuretics, calcium channel- or } \\
\text { alphal-blockers } \\
\text { Tissue RAAS may not be similarly suppressed (e.g., kidney) }\end{array}$ \\
\hline $\begin{array}{l}\text { Primary aldosteronism and other } \\
\text { MC excess }\end{array}$ & $\begin{array}{l}\text { Primary hypersecretion of aldosterone or } \\
\text { other MC hormone (e.g., DOC) by adrenal } \\
\text { neoplasms; or very rare genetic syndromes } \\
\text { resulting in MC hormone dysregulation } \\
\text { Secondary suppression of renin and Ang II }\end{array}$ & $\begin{array}{l}\text { Hypertension (may be severe at times) } \\
\text { Hypokalemia and hypomagnesemia } \\
\text { Sodium and volume expansion, without edema (limited by } \\
\text { "mineralocorticoid escape") } \\
\text { Reversible atrophy of contralateral adrenal cortex (due to suppressed } \\
\text { Ang II) }\end{array}$ \\
\hline
\end{tabular}

Ang = angiotensin aldosterone system; $D O C=$ deoxycorticosterone; $H R=$ high renin; $J G=$ juxtaglomerular; $L R=$ low renin; $M C=$ mineralocorticoid; $N R=$ normal renin; PRA = plasma renin activity; RAAS = renin-angiotensin aldosterone system. $A$ ? indicates that the true etiology is unknown. 


\section{Beta Blockers}

In the 1970s, Laragh and colleagues showed that, in patients with various types of hypertension, beta-blocker therapy (propranolol) reduced plasma renin levels by blocking sympathetically (beta $)_{1}$ )-mediated renin release by the kidney. ${ }^{26}$ They concluded that the reduction in plasma renin level (by about $75 \%$ ) was closely correlated with reductions in blood pressure. They also showed that propranolol reduced aldosterone secretion. Their results demonstrated the viability of pharmacologic blockade of the RAAS as a therapeutic strategy.

A more recent study demonstrated parallel suppression of Ang II and PRA during beta-blocker therapy in hypertensive subjects. ${ }^{27}$ In addition to suppression of renin release, there is evidence that beta blockade may also inhibit intrarenal conversion of prorenin to renin. ${ }^{27,28}$ The blood pressure-lowering activity of beta blockers occurs via both renin-dependent and renin-independent mechanisms. Although many studies suggest a preferential effect among patients with high-renin forms of hypertension, ${ }^{25,26}$ there is evidence for benefit in low-renin hypertension as well, particularly with high-dose propranolol therapy (320 mg to $980 \mathrm{mg}$ daily), independent of changes in PRA. ${ }^{29}$

\section{Angiotensin-Converting Enzyme Inhibitors}

Early studies performed in the 1960s showed that peptides from the venom of the Brazilian arrowhead viper (Bothrops jararaca) inhibited kinase II, an enzyme that facilitates degradation of bradykinin, and which was later shown to be identical to ACE. ${ }^{2}$ Synthetic analogues of the peptide fraction of snake venom, such as the nonapeptide teprotide, were shown to lower blood pressure in patients with hypertension and produce beneficial hemodynamic effects in patients with heart failure. ${ }^{2}$ These findings encouraged the search for orally active inhibitors of ACE; the first of these, captopril, was designed based on known inhibitors of another zinc-containing metalloprotease, carboxypeptidase A, and included a sulfhydryl-containing amino acid to serve as ligand for the zinc moiety. Because many of the unacceptable side effects of captopril, such as proteinuria, skin rashes, and altered taste, were attributed to the sulfhydryl group, subsequent work led to the development of ACEIs that replaced this group with a carboxyl group (e.g., lisinopril, benazepril, quinapril, ramipril, perindopril, cilazapril, trandolapril) or phosphoryl group (fosinopril). ${ }^{30,31}$ The presence of the carboxyl group conferred greater lipophilicity, which actually improved binding to ACE, and improved tissue penetration. ${ }^{31}$

ACEIs competitively block the action of ACE and thus the conversion of Ang I to Ang II, thereby reducing circulating and local levels of Ang II. ACEIs also decrease aldosterone and vasopressin secretion and sympathetic nerve activity, but there is controversy regarding their efficacy in blocking other "tissue" actions of the RAAS. ${ }^{32}$ Short-term ACEI therapy is associated with a decrease in Ang II and aldosterone and an increase in renin release and Ang I. There is some evidence, however, that over the long term ACE inhibition may be associated with a return of Ang II and aldosterone toward baseline levels ("ACE escape")-perhaps, it is proposed, through activation of the so-called alternate pathways (Figure). ${ }^{32,33}$ Undoubtedly this phenomenon has been greatly exaggerated, particularly from early studies using faulty methodology that did not specifically measure Ang II, and the relevance of alternate pathways of Ang II synthesis in the intact human is unclear at present. On the other hand, because ACEIs are all competitive inhibitors of the enzyme, it is possible that increased levels of Ang I (provoked by the compensatory increase in PRA due to loss of negative feedback inhibition) can tend to partially overcome the blockade. ${ }^{34}$ This would be especially likely in high-renin or volume-depleted patients with a particularly robust reactive rise in PRA.

In general, short-term, pharmacodynamic responses to decreases in Ang II through inhibition of ACE include dosedependent reductions in cardiac preload and afterload, with lowering of systolic and diastolic blood pressure, but, in normotensive and hypertensive patients without cardiac dysfunction, little or no change in cardiac output or capillary wedge pressure. Of note, unlike direct-acting arterial vasodilators, ACEI-induced reductions in total peripheral vascular resistance occur without a significant change in heart rate. ${ }^{32}$ ACEIs also decrease renal vascular resistance, increase renal blood flow, and promote sodium and water excretion. Mainly through cellular effects in the kidney and through alterations in glomerular hemodynamics, ACEIs also may prevent the progression of microalbuminuria to proteinuria, reduce proteinuria in patients with established glomerular disease, and prevent or delay the progression of renal insufficiency to end-stage renal disease. Efficacy in long-term trials has been demonstrated particularly in patients with nondiabetic nephropathies or in patients with insulin-dependent (type 1) diabetes. ${ }^{32,35,36}$

Because ACE is identical to kininase II, ACEIs may also lead to elevation of bradykinin levels in some tissues (but unlikely in the circulation); this effect is potentially associated with increased bradykinin-dependent release of $\mathrm{NO}$ and vasoactive prostaglandins, including prostacyclin and prostaglandin E2.32 These actions may potentially contribute to the vasodilatory, antithrombotic, antiatherogenic, and antiproliferative effects of ACEI, although the importance of this pathway is debated. ${ }^{32}$

In $40 \%$ to $60 \%$ of patients with mild-to-moderate hypertension, ACEI monotherapy produces a satisfactory reduction in blood pressure. ${ }^{37}$ In this population, ACEIs contribute to reversal of cardiac hypertrophy, and do so with significantly greater efficacy than beta blockers. ${ }^{38}$ In patients with CHF, ACEIs relieve pulmonary congestion by a balanced reduction in cardiac preload and afterload. They appear to induce venous vasodilation, which increases peripheral venous capacitance and reduces right atrial pressure, pulmonary arterial pressure, capillary wedge pressures, 
and left ventricular filling volumes and pressures. ACEIs also induce arterial vasodilation, which reduces peripheral vascular resistance (afterload) and increases cardiac output in this patient population. ${ }^{32}$ ACEIs have also been shown to improve endothelial dysfunction in patients with heart failure, as well as in patients with coronary artery disease and type 2 diabetes. ${ }^{32}$

In early landmark trials in patients with $\mathrm{CHF}$ (such as CONSENSUS, SOLVD, and V-HeFT-II), ACEIs were shown not only to markedly improve symptoms and functional status, but also to dramatically reduce mortality. In subsequent studies in patients who have suffered a myocardial infarction (MI), such as SAVE, AIRE, and TRACE, ACEI therapy has been shown to prevent or retard ventricular remodeling and progression to CHF, and thereby to reduce overall mortality and prolong survival. ${ }^{39-46}$ Furthermore, results of the HOPE trial and other smaller studies indicate broad cardiovascular benefits of ACEI therapy in "high-risk" patients (including both hypertensive and normotensive individuals), and it is possible that these benefits occur in part independently of their blood pressure-lowering effect. ${ }^{46}$

Several large-scale studies of various ACEIs have shown a reduction in incidence of new-onset diabetes in association with ACEI therapy. For example, this has been shown with captopril in patients with hypertension (CAPP) ${ }^{47}$ with ramipril in patients at high risk for cardiovascular disease (HOPE) ${ }^{46}$ with enalapril in patients with left ventricular dysfunction (SOLVD), ${ }^{48}$ and with trandolapril in patients with stable coronary disease (PEACE). ${ }^{49}$ The mechanism of this benefit has not been determined.

ACEI therapy is generally well tolerated by most patients but is nonetheless associated with some significant side effects. Most frequent among these is a dry cough, which has been attributed to accumulation of substance P (which is normally degraded by kininase II). More serious side effects common to all ACEIs include angioedema (which is potentiated by decreased catabolism of kinins) and fetal abnormalities and mortality. Other "physiologic" consequences of ACE inhibition may include hypotension, deterioration of renal function, and hyperkalemia. Lastly, toxic effects, associated mainly with captopril, include abnormal (metallic or salty) taste, rash, neutropenia, hepatic toxicity, and proteinuria (membranous nephropathy). ${ }^{30}$

\section{Angiotensin Receptor Blockers (ARBs)}

As mentioned earlier, the $\mathrm{AT}_{1}$ receptor mediates most of the known actions of Ang II that contribute to hypertension and volume dysregulation (vascular smooth muscle contraction, aldosterone secretion, dipsogenic responses, renal sodium reabsorption, and pressor and tachycardiac responses) as well as to cardiovascular damage (cellular hypertrophy or proliferation, prothrombotic and proinflammatory effects, and superoxide formation). ${ }^{7,9}$ Thus, with the discovery of different receptor subtypes, specific antagonism of Ang II action at the $\mathrm{AT}_{1}$ receptor became a logical therapeutic target, one considered likely to be more specific than ACE inhibition. Development of orally active, nonpeptide, selective $\mathrm{AT}_{1}$ receptor blockers began in the 1990s with the synthesis of losartan. ${ }^{2}$ Since that time, several ARBs have been synthesized, including valsartan, irbesartan, candesartan, eprosartan, telmisartan, and olmesartan.

Because ARBs act by blocking Ang II action at the receptor level, rather than by inhibiting its synthesis, they ought to antagonize $\mathrm{AT}_{1}$-mediated effects of Ang II no matter how it is synthesized. In other words, if there were significant Ang II synthesis in tissues by alternate pathways, such as chymase in the heart, this would limit the efficacy of ACEIs (but not of ARBs) through a mechanism postulated to contribute to the "escape" phenomenon following long-term ACE inhibition. ${ }^{50}$

In contrast to the ACEIs, ARB therapy actually results in an increase in Ang II levels. ${ }^{51}$ As with ACE inhibition, blockade of the $\mathrm{AT}_{1}$ receptor inhibits the negative feedback loop, leading to increased renin secretion and thus to increased synthesis of Ang I. In the case of ARBs, the increase in Ang I leads to a commensurate increase in Ang II, which is freely able to bind to $\mathrm{AT}_{2}$ or other receptor subtypes. Earlier preclinical studies have suggested that beyond $\mathrm{AT}_{1}$ receptor blockade, activation of the $\mathrm{AT}_{2}$ receptor might mediate additional beneficial actions on the vasculature, heart, and kidneys, in part via a bradykinin/NO/ cGMP pathway, an effect that would further distinguish ARBs from ACEIs. ${ }^{7}$ But as attractive as this hypothesis is, there are no clinical data to indicate that this pathway is a major mechanism of ARB action in humans.

Like the ACEIs, ARBs reduce blood pressure by decreasing systemic vascular resistance; they do not affect heart rate and have minimal effect on cardiac output in the nonfailing heart. ${ }^{51}$ Reduced systemic vascular resistance results from a combination of inhibition of Ang II-mediated vasoconstriction, reduced sympathetic nervous system activity, and reduced extracellular volume (i.e., by direct inhibition of proximal sodium reabsorption and by inhibition of aldosterone release). ${ }^{51}$ ARB monotherapy produces a satisfactory reduction in blood pressure in $40 \%$ to $60 \%$ of patients with mild-to-moderate hypertension. ${ }^{37}$ ARB therapy has also been shown to reduce markers of inflammation in patients with atherosclerosis, ${ }^{52,53}$ suggesting an anti-inflammatory effect, and to reverse endothelial dysfunction in patients with hypertension, indicating the possibility of significant antiatherogenic effects. ${ }^{53}$ In patients with hypertension, ARB therapy has also been shown to improve arterial compliance independent of the blood pressure-lowering effect. This observation suggests that ARB therapy may contribute to reversal of vascular wall damage..$^{54}$

A number of recent large trials support the idea that the ARB class may confer benefits on target organ protection beyond the lowering of blood pressure per se. In patients with hypertension and left ventricular hypertrophy, ARB-based therapy, compared with beta-blocker (atenolol)-based therapy with identical blood pressure control, has been shown to significantly reduce the 
composite risk of cardiovascular death, stroke, and MI and to decrease the rate of new-onset diabetes (LIFE study). ${ }^{55}$ Similarly, ARB-based therapeutic regimens, compared with conventional therapy, have been shown to reduce the progression of nephropathy in patients with diabetic nephropathy (IDNT, RENAAL studies). ${ }^{56,57}$ In nonhypertensive conditions, ARBs have shown benefits comparable with those of ACEIs. In patients with chronic heart failure, addition of an ARB, compared with placebo, to conventional treatment has been shown to significantly reduce the risk of cardiovascular mortality and hospitalization (CHARM, Val-HeFT studies). ${ }^{58,59}$ In high-risk post-MI patients, ARB therapy has been shown to reduce the risks of all-cause mortality, recurrent MI, sudden cardiac death, revascularization, coronary artery bypass grafting, or all-cause hospital admission to a degree similar to that of ACEI therapy (OPTIMAAL study). ${ }^{60}$

Like ACEIs, use of ARBs is contraindicated in pregnant women because of the association of RAAS blockade with increased fetal morbidity and mortality, particularly with exposure during the second and third trimester. ARB therapy is otherwise generally well tolerated, even in many patients who discontinue ACEI therapy because of side effects. Although rare reports of angioedema and cough have emerged from the early premarketing clinical trials with ARBs, it is currently debated whether these are truly associated with the class as they are with ACEIs. Most adverse events reported with ARB therapy are related to expected potential effects of RAAS blockade-for example, hypotension, hyperkalemia, and worsening renal function-and are similar to those encountered in patients taking ACEIs.

\section{Direct Renin Inhibitors}

The most recent class of agents that block the RAAS to be introduced are the direct renin inhibitors represented by aliskiren, which was recently approved for treatment of hypertension. This compound differs from the ACEIs and ARBs in that, by blocking the catalytic activity of renin at the point of activation of the RAAS, it blocks the synthesis of all angiotensin peptides and prevents the compensatory increase in renin activity. This topic is dealt with in detail in another article in this series (see article titled "Direct Renin Inhibition: Focus on Aliskiren" by Pool). ${ }^{61}$

\section{Future Directions}

ACEIs and ARBs are currently indicated for the treatment of hypertension, diabetic nephropathy, post-MI left ventricular dysfunction, and chronic heart failure, and their use has been associated with improved survival and considerable cardiovascular and renal benefits in high-risk patients. These remarkable benefits have been obtained even though blockade of the RAAS with currently available agents may be incomplete, raising the possibility that additional therapeutic modalities for RAAS blockade might help to further slow progression of cardiovascular and renal disease.
First, blockade of the RAAS by ACEIs and ARBs is incomplete because their therapeutic response can be limited by the reactive rise in PRA. This is particularly so with the ACEIs, because a marked rise in Ang I may compete with the relatively lowaffinity inhibition of ACE that they afford. Second, although the notion that selective blockade of $\mathrm{AT}_{1}$ receptors may be a preferred approach over ACE inhibition is based on attractive hypotheses (i.e., the potential benefits of $\mathrm{AT}_{2}$ receptor agonism and/or the ability to counteract the effects of ACE-independent pathways of Ang II synthesis), these hypotheses are largely unproven in humans. Moreover, there have been no large-scale clinical trials over the past 15 years that demonstrate a clear superiority of ARBs over ACEIs. Third, although our focus has been on Ang II as the only villain of the RAAS, the possibility that other angiotensin peptides might also contribute to cardiovascular pathology has never been adequately tested. Fourth, questions of mechanism aside, it is likely that differences in drug distribution and/or tissue penetration between or within the classes may limit the benefits of any single agent. Thus, it is reasonable at this point to ask whether more-complete blockade of the system will offer even more clinical benefits.

The clinical potential of simultaneous intervention at multiple sites of the RAAS is compelling. As has already been demonstrated in small clinical trials, dual RAAS blockade using an ACEI and ARB in combination may have potential clinical value in symptomatic patients with $\mathrm{CHF}$ or left ventricular systolic dysfunction and in patients with chronic proteinuric renal disease. ${ }^{50}$ With the recent availability of aliskiren, the first direct renin inhibitor approved for the treatment of hypertension, the opportunity is at hand to test new therapeutic approaches involving monotherapy with this agent or its use in combination with an ACEI or ARB for more complete blockade of the RAAS. Results obtained with this approach may direct investigators to explore additional therapeutic targets in the future, such as other angiotensin receptor subtypes, other relatively specific metabolic pathways (e.g., "ACE 2"), or other angiotensin peptides (e.g., Ang III, Ang IV, and Ang-(1-7)). Lastly, the possibility of molecular approaches such as antisense gene therapy, targeting, for instance, renin, angiotensinogen, the $\mathrm{AT}_{1}$ receptor, or $\mathrm{ACE}$, will also likely be explored in the not-too-distant future.

\section{DISCLOSURES}

The author discloses no potential bias or conflict of interest relating to this article.

\section{REFERENCES}

1. Piepho RW, Beal J. An overview of antihypertensive therapy in the 20th century. J Clin Pharmacol. 2000;40:967-77.

2. Ferrario CM. Role of angiotensin II in cardiovascular diseasetherapeutic implications of more than a century of research.

J Renin Angiotensin Aldosterone Syst. 2006;7:3-14. 
3. Skeggs LT Jr, Kahn JR, Lentz K, Shumway NP. The preparation, purification, and amino acid sequence of a polypeptide renin substrate. J Exp Med. 1957;106:439-53.

4. Sealey JE, Laragh JH. Renin-angiotensin-aldosterone system and the renal regulation of sodium, potassium, and pressure homeostasis. In: Windhager EE, ed. Handbook of Physiology-Section 8: Renal Physiology. Vol II. New York: Oxford University Press; 1992:1409-541.

5. Brown MJ. Direct renin inhibition-a new way of targeting the renin system. J Renin Angiotensin Aldosterone Syst. 2006;7(suppl 2):S7-S11.

6. Morgan L, Broughton PF, Kalsheker N. Angiotensinogen: molecular biology, biochemistry and physiology. Int J Biochem Cell Biol. 1996;28:1211-22

7. Carey RM, Siragy HM. Newly recognized components of the renin-angiotensin system: potential roles in cardiovascular and renal regulation. Endocr Rev. 2003;24:261-71.

8. Reudelhuber TL. The renin-angiotensin system: peptides and enzymes beyond angiotensin II. Curr Opin Nephrol Hypertens. 2005;14:155-59.

9. Stanton A. Therapeutic potential of renin inhibitors in the management of cardiovascular disorders. Am J Cardiovasc Drugs. 2003;3:389-94.

10. Nguyen G, Delarue F, Burckle C, Bouzhir L, Giller T, Sraer J-D Pivotal role of the renin/prorenin receptor in angiotensin II production and cellular responses to renin. J Clin Invest. 2002;109:1417-27.

11. Ichihara A, Hayashi M, Kaneshiro Y, et al. Inhibition of diabetic nephropathy by a decoy peptide corresponding to the "handle" region for nonproteolytic activation of prorenin. J Clin Invest. 2004;114:1128-35.

12. Funder JW. New biology of aldosterone, and experimental studies on the selective aldosterone blocker eplerenone. Am Heart J. 2002;144:S8-11.

13. Phillips MI. Tissue renin-angiotensin systems. In: Izzo JL, Black HR, ed. Hypertension Primer: The Essentials of High Blood Pressure. 2nd ed. Baltimore, MD: Lippincott William \& Wilkins; 1999:23-24

14. Ihara M, Urata H, Kinoshita A, et al. Increased chymase-dependent angiotensin II formation in human atherosclerotic aorta. Hypertension. 1999;33:1399-405.

15. Hollenberg NK, Fisher NDL, Price DA. Pathways for angiotensin II generation in intact human tissue: evidence from comparative pharmacological interruption of the renin system. Hypertension. 1998;32:387-92.

16. Urata H, Healy B, Stewart RW, Bumpus FM, Husain A. Angiotensin II-forming pathways in normal and failing human hearts. Circ Res. 1990;66:883-90.

17. Wolny A, Clozel JP, Rein J, et al. Functional and biochemical analysis of angiotensin II-forming pathways in the human heart. Circ Res. 1997:80:219-27.

18. Pagliaro P, Penna C. Rethinking the renin-angiotensin system and its role in cardiovascular regulation. Cardiovasc Drugs Ther. 2005;19:77-87.

19. Paul M, Poyan Mehr A, Kreutz R. Physiology of local renin-angiotensin systems. Physiol Rev. 2006;86:747-803.

20. Danser AH, van Kats JP, Admiraal PJ, et al. Cardiac renin and angiotensins. Uptake from plasma versus in situ synthesis. Hypertension. 1994:24:37-48

21. van Kats JP, Chai W, Duncker DJ, Schalekamp MADH, Danser AHJ. Adrenal angiotensin: origin and site of generation. Am J Hypertens. 2005;18:1104-10

22. Laragh J. Laragh's lessons in pathophysiology and clinical pearls for treating hypertension. Am J Hypertens. 2001;14:186-94.

23. Schmidt RJ, Soman SS. Renovascular Hypertension. Available at: http://www.eMedicine.com. Accessed May 3, 2007.

24. Corvol P, Pinet F, Plouin PF, Bruneval P, Menard J. Renin-secreting tumors. Endocrinol Metab Clin North Am. 1994;23:255-70.
25. Staessen JA, Li Y, Richart T. Oral renin inhibitors. Lancet. 2006;368:1449-56.

26. Laragh J. Laragh's lessons in pathophysiology and clinical pearls for treating hypertension. Am J Hypertens. 2001;14:296-304.

27. Blumenfeld JD, Sealey JE, Mann SJ, et al. $\beta$-Adrenergic receptor blockade as a therapeutic approach for suppressing the renin-angiotensinaldosterone system in normotensive and hypertensive subjects. Am J Hypertens. 1999;12:451-59.

28. Atlas SA, Sealey JE, Laragh JH, Moon C. Plasma renin and "prorenin" in essential hypertension during sodium depletion, beta-blockade, and reduced arterial pressure. Lancet. 1977;2:785-89.

29. Hollifield JW, Sherman K, Zwagg RV, Shand DG. Proposed mechanisms of propranolol's antihypertensive effect in essential hypertension.

N Engl J Med. 1976;295:68-73.

30. Wong J, Patel RA, Kowey PR. The clinical use of angiotensin-converting enzyme inhibitors. Prog Cardiovasc Dis. 2004:47:116-30.

31. Brown B, Hall AS. Renin-angiotensin system modulation: the weight of evidence. Am J Hypertens. 2005;18(9 pt 2):127S-33S.

32. Lopez-Sendon J, Swedberg K, McMurray J, et al., for the Task Force on ACE-inhibitors of the European Society of Cardiology. Expert consensus document on angiotensin converting enzyme inhibitors in cardiovascular disease. Eur Heart J. 2004;25:1454-70.

33. Pitt B. "Escape" of aldosterone production in patients with left ventricular dysfunction treated with an angiotensin converting enzyme inhibitor: implications for therapy. Cardiovasc Drugs Ther. 1995;9:145-49.

34. Atlas SA, Case DB, Yu ZY, Laragh JH. Hormonal and metabolic effects of angiotensin converting enzyme inhibitors: possible differences between enalapril and captopril. Am J Med. 1984;77:13-17.

35. Maschio G, Alberti D, Janin G, et al. Effect of the angiotensinconverting-enzyme inhibitor benazepril on the progression of chronic renal insufficiency. N Engl J Med. 1996;334:939-45.

36. Lewis EJ, Hunsicker LG, Bain RP, Rohde RD, for the collaborative study group. The effect of angiotensin-converting-enzyme inhibition on diabetic nephropathy. N Engl J Med. 1993;329:1456-62.

37. Ibrahim MM. RAS inhibition in hypertension. J Hum Hypertens. 2006;20:101-08.

38. Klingbeil AU, Schneider M, Martus P, Messerli FH, Schmieder RE. A meta-analysis of the effects of treatment on left ventricular mass in essential hypertension. Am J Med. 2003;115:41-46.

39. The SOLVD Investigators. Effect of enalapril on mortality and the development of heart failure in asymptomatic patients with reduced left ventricular ejection fractions. N Engl J Med. 1992;327:685-91.

40. Effect of enalapril on survival in patients with reduced left ventricular ejection fractions and congestive heart failure. The SOLVD Investigators. N Engl J Med. 1991;325:293-302.

41. Gruppo Italiano per lo Studio della Sopravvivenza nell'infarto Miocardico. GISSI-3: effects of lisinopril and transdermal glyceryl trinitrate singly and together on 6-week mortality and ventricular function after acute myocardial infarction. Lancet. 1994;343:1115-22.

42. ISIS-4 (Fourth International Study of Infarct Survival) Collaborative Group. ISIS-4: a randomised factorial trial assessing early oral captopril, oral mononitrate, and intravenous magnesium sulphate in 58,050 patients with suspected acute myocardial infarction. Lancet. 1995;345:669-85.

43. Pfeffer MA, Braunwald E, Moye LA, et al. Effect of captopril on mortality and morbidity in patients with left ventricular dysfunction after myocardial infarction. Results of the survival and ventricular enlargement trial. The SAVE Investigators. N Engl J Med. 1992;327:669-77. 
44. The Acute Infarction Ramipril Efficacy (AIRE) Study Investigators. Effect of ramipril on mortality and morbidity of survivors of acute myocardial infarction with clinical evidence of heart failure. Lancet. 1993;342:821-28.

45. The EURopean trial On reduction of cardiac events with Perindopril in stable coronary Artery disease [EUROPA] Investigators. Efficacy of perindopril in reduction of cardiovascular events among patients with stable coronary artery disease: randomised, double-blind, placebo-controlled, multicentre trial (the EUROPA study). Lancet. 2003;362:782-88.

46. Yusuf S, Sleight P, Pogue J, et al., for The Heart Outcomes Prevention Evaluation Study Investigators. Effects of an angiotensin-converting-enzyme inhibitor, ramipril, on cardiovascular events in high-risk patients.

N Engl J Med. 2000;342:145-53.

47. Hansson L, Lindholm LH, Niskanen L, et al. Effect of angiotensinconverting-enzyme inhibition compared with conventional therapy on cardiovascular morbidity and mortality in hypertension: the Captopril Prevention Project (CAPPP) randomised trial. Lancet. 1999;353:611-16.

48. Vermes E, Ducharme A, Bourassa MG, Lessard M, White M, Tardif J-C. Enalapril reduces the incidence of diabetes in patients with chronic heart failure: insight from the Studies Of Left Ventricular Dysfunction (SOLVD). Circulation. 2003;107:1291-96.

49. The PEACE Trial Investigators. Angiotensin-converting-enzyme inhibition in stable coronary artery disease. N Engl J Med. 2004:351:2058-68.

50. Ruilope LM, Rosei EA, Bakris GL, et al. Angiotensin receptor blockers: therapeutic targets and cardiovascular protection. Blood Press. 2005;14:196-209.

51. Hernandez-Hernandez R, Sosa-Canache B, Velasco M, Armas-Hernandez MJ, Armas-Padilla MC, Cammarata R. Angiotensin II receptor antagonists role in arterial hypertension. J Hum Hypertens. 2002;16(suppl 1):S93-S99.

52. Navalkar S, Parthasarathy S, Santanam N, Khan BV. Irbesartan, an angiotensin type 1 receptor inhibitor, regulates markers of inflammation in patients with premature atherosclerosis. J Am Coll Cardiol. 2001;37:440-44.
53. Koh KK, Ahn JY, Han SH, et al. Pleiotropic effects of angiotensin II receptor blocker in hypertensive patients. J Am Coll Cardiol. 2003;42:905-10.

54. Shargorodsky M, Leibovitz E, Lubimov L, Gavish D, Zimlichman R. Prolonged treatment with the $\mathrm{AT}_{1}$ receptor blocker, valsartan, increases small and large artery compliance in uncomplicated essential hypertension. Am J Hypertens. 2002;15:1087-91.

55. Dahlof B, Devereux RB, Kjeldsen SE, et al. Cardiovascular morbidity and mortality in the Losartan Intervention For Endpoint reduction in hypertension study (LIFE): a randomised trial against atenolol. Lancet. 2002:359:995-1003.

56. Lewis EJ, Hunsicker LG, Clarke WR, et al. Renoprotective effect of the angiotensin-receptor antagonist irbesartan in patients with nephropathy due to type 2 diabetes. N Engl J Med. 2001;345:851-60.

57. Brenner BM, Cooper ME, De Zeeuw D, et al. Effects of losartan on renal and cardiovascular outcomes in patients with type 2 diabetes and nephropathy. N Engl J Med. 2001;345:861-69.

58. Pfeffer MA, Swedberg K, Granger CB, et al. Effects of candesartan on mortality and morbidity in patients with chronic heart failure: the CHARM-Overall programme. Lancet. 2003;362:759-66.

59. Maggioni AP, Anand I, Gottlieb SO, et al. Effects of valsartan on morbidity and mortality in patients with heart failure not receiving angiotensin-converting enzyme inhibitors. J Am Coll Cardiol. 2002;40:1414-21

60. Dickstein K, Kjekshus J. Effects of losartan and captopril on mortality and morbidity in high-risk patients after acute myocardial infarction: the OPTIMAAL randomised trial. Optimal Trial in Myocardial Infarction with Angiotensin II Antagonist Losartan. Lancet. 2002;360:752-60.

61. Pool JL. Direct renin inhibition: focus on aliskiren. J Manag Care Pharm. 2007;13(8)(suppl b):S21-S33. 